\title{
Programa de deshabituación tabáquica para internos y trabajadores de un centro penitenciario
}

\author{
C Yagüe-Olmos ${ }^{1}$, MI Cabello-Vázquez²
}

${ }^{1}$ Directora del Centro Penitenciario de Mujeres de Alcalá de Guadaíra (Sevilla)

${ }^{2}$ Subdirectora de Tratamiento

\section{RESUMEN}

El principal objetivo de esta iniciativa es promover un cambio de cultura en torno al binomio cárcel y tabaco mediante un programa de intervención que conduzca a una reducción del elevado porcentaje de tabaquismo entre la población interna, 70 a $80 \%$, y los trabajadores del medio penitenciario.

Queremos demostrar, en la práctica, que es posible implantar y generalizar con éxito programas de abandono de la dependencia del tabaco en los Establecimientos penitenciarios. Esto supone un paso importante en la mejora de la salud global de los internos que sufren una mayor prevalencia de tabaquismo que la población general y de los trabajadores penitenciarios, que comparten con ellos un espacio reducido y cerrado.

Palabras clave: Tabaquismo, Programas de Abandono del Tabaco.

\section{A SMOKING CESSATION PROGRAM FOR PRISON INMATES AND PERSONNEL}

\begin{abstract}
The main aim of this initiative is to promote a culture change in the prison/tobacco conjunction by means of an intervention program that leads to reduction in the high levels of smoking amongst the prison population (70-80\%) and prison personnel.

We hope to demonstrate that it is possible in practice to successfully implant and extend tobacco cessations programs in prisons. This would mean an important step forward for the general health of prison inmates, who suffer from higher levels of tobacco addiction than the general population and prison staff, who for their part are forced to share a reduced and enclosed space with prisoners.
\end{abstract}

Key Words: Tobacco Addiction, Tobacco Cessations Programs.

Texto recibido: abril 2008

Texto aceptado: junio 2008

\section{INTRODUCCIÓN}

El hábito del tabaquismo, bastante extendido en la población general, aumenta significativamente en situaciones de estrés y privaciones. En el ámbito carcelario, el consumo de tabaco se ha asociado históricamente a las consecuencias negativas de la pérdida de libertad y a los conflictos personales y situacionales subyacentes a la misma.
Consecuentemente, los problemas de salud pública que acarrea este hábito se hacen sentir marcadamente en la población reclusa, cuyo porcentaje de fumadores duplica al de la población libre.

Además, en las prisiones predominan los espacios cerrados y coexisten con una fuerte limitación de movimientos lo que incide de forma muy particular en los reclusos no fumadores que se ven obligados a compartir estos locales sin apenas capacidad de sus- 
traerse a sus efectos nocivos. Incluso han de convivir con fumadores muchas horas del día, a veces, incluso en el propio dormitorio o espacio residencial sin contar con una adecuada ventilación. Estas limitaciones afectan, de similar manera, a los trabajadores penitenciarios que comparten largas jornadas en este ambiente.

La Ley de tabaquismo que entró en vigor en enero de 2006 ha supuesto un punto de inflexión importante, al limitar radicalmente los espacios comunes habilitados para fumar, aunque se permite hacerlo en las habitaciones, que tienen consideración de domicilio propio.

Un año antes de su implantación en el Centro Penitenciario de Mujeres de Alcalá de Guadaíra nos planteamos que esta circunstancia suponía una excelente oportunidad para promover un cambio de cultura en torno al binomio cárcel y tabaco mediante un programa de intervención que conduzca a una reducción del elevado porcentaje de tabaquismo entre la población interna (que se sitúa entre el 70-80\% en este centro ${ }^{1}$ ) y los trabajadores del medio penitenciario.

Esta iniciativa surge dentro del programa de prevención de salud de este centro, y como una experiencia pionera dentro de los Establecimientos Penitenciarios. Los profesionales del centro carecíamos de una adecuada preparación para acometer estos programas, por lo que acudimos al servicio público de salud de nuestra Comunidad Autónoma. En un primer momento se obtuvo la entusiasta participación del personal del Servicio de Deshabituación tabáquica del Hospital de Valme, que contaba con una gran experiencia, y con los que acometimos la primera edición del programa desarrollada entre 2005-2006. Simultáneamente se formalizó la colaboración con los responsables de la Consejería de Sanidad, que se fraguó en la segunda edición para la que se contó con la colaboración del Distrito Sanitario Sur de Alcalá de Guadaíra. Todo ello enmarcado dentro del Plan Integral de Atención al Tabaquismo (2005-2010) de la Junta de Andalucía. Nace así una colaboración interinstitucional cuyo programa, método y resultados presentamos en el presente artículo.

\section{ANÁLISIS SITUACIONAL}

El Centro Penitenciario de Alcalá de Guadaíra (Sevilla) se creó en el año 1991 para atender la creciente demanda de plazas para mujeres presas en la Comunidad Autónoma de Andalucía y albergar, además,

\footnotetext{
${ }^{1}$ Según encuesta previa realizada al inicio de la experiencia.
}

dentro de él la primera Unidad para internas madres con sus hijos de esta región.

Su capacidad máxima es de 220 plazas, sin embargo, ordinariamente, su ocupación real se encuentra muy por debajo de estas cifras. Actualmente, residen en él una media de 160 internas, de las cuales el $98 \%$ están condenadas. 20 internas $(12,5 \%)$ residen en la Unidad Maternal con sus hijos menores de tres años. 15 internas (el 9,3\%) disfrutan de régimen de semilibertad en la sección abierta, en la Unidad Dependiente de madres, o bajo el control de medios telemáticos.

El perfil medio de una interna de este Establecimiento sería una mujer de 30-38 años, residente de una barriada marginal de Sevilla o Málaga, con un nivel escolar muy bajo (analfabetismo funcional o estudios básicos). Un alto porcentaje, el $62 \%$ son politoxicómanas; se encuentran infectadas por VIH el $7 \%$ de la población y por VHC el $22 \%$; toma algún tipo de psicofármaco el $34 \%$, y están en programa de mantenimiento con metadona (PMM) el 14\%.

\section{POBLACIÓN A QUIEN SE DIRIGE}

El programa se ofrece a la totalidad de internas del centro que deseen deshabituarse del tabaco. Entre las que solicitan su participación se excluyen a aquellas que, a través de las entrevistas iniciales multidisciplinares, no manifiestan una motivación adecuada, y a quienes se prevé que no pueden permanecer en el centro el tiempo suficiente para completar el programa por su situación penitenciaria.

Se dirige también a los trabajadores del centro con similares características. Se selecciona exclusivamente a los que asumen el compromiso expreso de asistir a todas las actividades grupales conjuntas, aun cuando esto les suponga el desplazamiento al centro en días no coincidentes con su turno de trabajo.

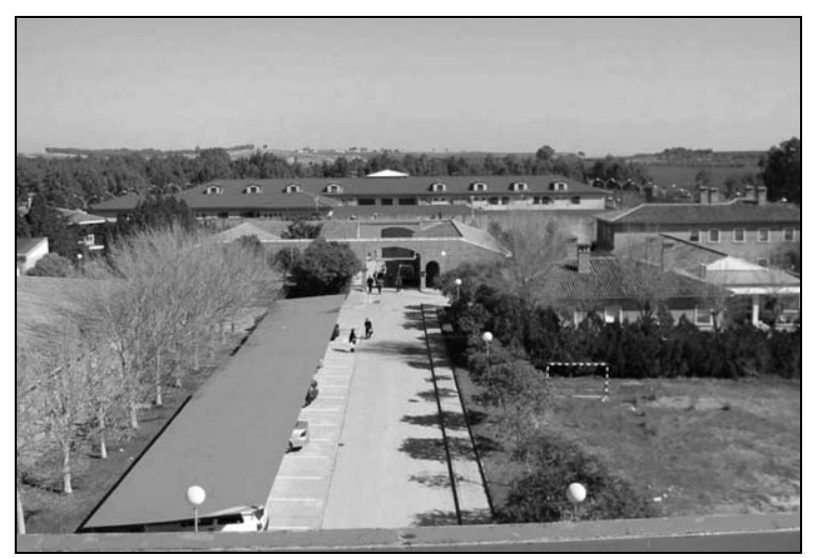


El grupo definitivo de intervención lo componen un número aproximado de 20-22 internas y 3-5 trabajadores.

\section{OBJETIVOS DE ACTUACIÓN}

\section{Objetivos específicos:}

1. Contribuir a reducir los problemas de salud asociados a un consumo habitual del tabaco.

2. Promover hábitos saludables que mejoren el bienestar general de las internas y los profesionales del centro.

3. Contribuir a facilitar la convivencia entre las internas, y entre éstas y los trabajadores del centro, respetando que la mayoría de los espacios que se encuentran obligados a compartir sean espacios libres de humo.

4. Contribuir a reducir el impacto monetario que supone el consumo de tabaco sobre la precaria economía de las internas fumadoras, y reducir la frecuente dependencia económica de algunas de ellas, de las más pudientes, lo que las hace susceptibles de padecer situaciones de abusos y humillaciones.

5. Contribuir a un cambio de cultura respecto a la inocuidad del tabaco y la permisividad hacia el fumador en espacios penitenciarios.

6. Contribuir a la toma de conciencia de que el tabaco es una droga.

7. Contribuir a un mayor conocimiento de los efectos nocivos del tabaco sobre el organismo.

8. Contribuir a fortalecer la voluntad, la autonomía y la autoestima de los componentes del grupo que participa en la intervención.

\section{Evaluación de necesidades}

Con el objeto de conocer las características de la población potencial sobre la que se iba a realizar la intervención, y permitir el desarrollo de indicadores para la posterior evaluación del mismo, se llevó a cabo una encuesta previa al inicio del primer programa. Se contaba además, como referencia, con otra encuesta realizada en 2004, en el medio extrapenitenciario sobre una población estadísticamente significativa de mujeres de 15 a 55 años de la provincia de Sevilla sobre hábitos, actitudes y conocimientos sobre el consumo de tabaco ${ }^{2}$. La encuesta realizada en el medio

\footnotetext{
${ }^{2}$ Realizada por personal del Hospital Valme "Hábitos, actitudes y conocimientos sobre el consumo de tabaco en las mujeres de 15 a 55 años en la Provincia de Sevilla”. 2004. Documento no publicado.
}

penitenciario se llevó a cabo en julio de 2005 abarcando a la totalidad de internas del centro (160 internas, de las que fueron contestados 117 cuestionarios, es decir, el 73\%).

Los principales resultados (comparando los datos obtenidos en ambas encuestas) son los siguientes:

- Se declaran fumadoras el $81,5 \%$ de las internas encuestadas en este centro penitenciario, frente al $39,4 \%$ de mujeres encuestadas en la provincia de Sevilla ${ }^{3}$. Por lo tanto, el porcentaje de fumadoras en prisión duplica al de fumadoras en el medio libre. El porcentaje de fumadoras en nuestro centro es similar al encontrado en la Encuesta de Salud de Instituciones Penitenciarias que alcanza el 73,1\% ${ }^{4}$.

- El consumo diario de cigarrillos en nuestra prisión es de 20,3, frente al 13,7 observado en el medio extrapenitenciario ${ }^{5}$.

- El gasto medio estimado en tabaco por interna/día es de $2,38 €$.

- Un 44\% de las internas declaran que su consumo de cigarrillos en prisión ha aumentado con respecto a lo que consumían previamente a su ingreso en prisión.

- Tras las primeras sesiones de sensibilización, el 86\% de las internas expresaron su deseo de abandono del tabaco y el $65 \%$ de ellas manifiestan haber hecho algún intento previo de abandono. Estos datos son similares a los encontrados en la bibliografía nacional e internacional ${ }^{6}$.

- Respecto a los 140 trabajadores del centro penitenciario, contamos con los resultados de los reconocimientos médicos de empresa que se realizan con carácter voluntario cada año. En la revisión de octubre de 2005 , en la que se sometieron a reconocimiento 60 trabajadores (el 42,8\% de los empleados del centro penitenciario), se declara fumador el $23 \%$.

\section{METODOLOGÍA DE LA INTERVENCIÓN}

En primer lugar se llevó a cabo una etapa previa de estrecha colaboración intersectorial entre el medio penitenciario y el medio sanitario que permitió el di-

\footnotetext{
${ }^{3}$ Ídem nota 2.

${ }^{4}$ Encuesta de salud de instituciones penitenciarias 2006. Datos aportados por la Delegación del Gobierno para el Plan Nacional sobre Drogas.

${ }^{5}$ Ídem nota 2.

${ }^{6}$ Sieminska A. Prisoners'attitudes towards cigarette smoking and smoking cessation: a questionnarire study in Poland. BMC Public Health 2006 jul 7; 6: 181; y encuesta de población externa.
} 
seño ajustado de la intervención. Se mantuvieron diversas reuniones con objeto de definir actividades, repartir responsabilidades, acordar la formación y el entrenamiento del personal y establecer el calendario de cada actuación:

A) Entrenamiento del personal sanitario de la prisión en la Unidad de Atención al Tabaquismo de Valme (junio 2005).

B) Etapa de concienciación e información general, realización de las encuestas de consumo en la prisión, recogida de solicitudes de participación y selección del grupo de intervención (duración aproximada, 2 meses).

C) Aplicación a cada uno de los seleccionados el test de Fargerstron para evaluar el grado de dependencia a la nicotina.

D) Intervención con terapia multicomponente ${ }^{7}(\mathrm{du}-$ ración aproximada 4 meses). Implica la utilización interrelacionados de varios métodos:

- Terapia grupal:

- Consta de 10 sesiones formales ${ }^{8}$, de dos horas de duración dirigidas por los técnicos externos ${ }^{9}$ y en concurso con los trabajadores /terapeutas del centro. Comenzando por dos reuniones semanales, su frecuencia va espaciándose paulatinamente en el tiempo. En las sesiones de grupo se hacen rondas de participación donde cada componente del grupo comenta su evolución y analiza los detonantes del consumo, si lo hubiera, así como qué personas, situaciones o estrategias ayudan a mantener la abstinencia.

- En estas reuniones grupales se trabajan:

- Los beneficios de dejar de fumar.

- Plan individual para dejar de fumar.

- Estrategias para afrontar el día “D”, o día elegido para dejar de fumar.

- Afrontamiento de los síntomas del síndrome de abstinencia (craving -o deseo de fu-

\footnotetext{
${ }^{7}$ Stead, L.F., y Lancaster, T. (2002/2005). Group behaviour therapy programmes for smoking cessation (Cochrane Review). En The Cochrane Library, Issue 2. Oxford: Update Software.

${ }^{8}$ Mateos Vilchez P. Tratamiento grupal multicomponente. Red de Formadores del Plan Integral de Atención al Tabaquismo de Andalucía. www.redformadorespita.org

${ }^{9}$ Luis Lara (neumólogo) y José Ignacio Villar (psicólogo), técnicos de la Unidad de Deshabituación Tabáquica del Hospital de Valme, en su primera edicción, y Carmen Carmona (médico) y Reyes Sagrera (psicóloga), del área de salud del distrito sanitario de Alcalá de Guadaíra, coordinados por Manuel Cenizo en la segunda edición. A todos ellos les debemos nuestro más sincero agradecimiento por compartir con nosotros su formación y por su labor desinteresada.
}

mar-, ansiedad, irritabilidad, aumento de peso, frustración, melancolía, insomnio, etc.).

- Falsas creencias acerca del tabaco.

- Prevención de recaídas.

- Elementos situacionales que favorecen o torpedean el dejar de fumar.

- Entre estas reuniones formales, y a demanda del grupo, se intercalan sesiones de apoyo con el personal interno de la institución. Estas sesiones, de similar contenido, tienen un carácter de refuerzo de la abstinencia cuando por razones de calendario no pueden desplazarse los técnicos externos, o cuando existen circunstancias especiales, ya sean acontecimientos generales del centro, o problemas particulares de alguno de los componentes y se detecta la necesidad de un apoyo psicológico y grupal para prevenir recaídas.

- Medición en cada sesión del CO en aire expirado mediante cooximetría ${ }^{10}$ (sistema de feedback utilizado tanto en las que poseen carácter formal como las de apoyo). El cooxímetro es un aparato que permite el conocimiento de los niveles de $\mathrm{CO}$, de tal manera que a un mayor número de cigarrillos le corresponde unos más altos niveles de $\mathrm{CO}$ en el aire espirado. Es un método muy sencillo y eficaz para conocer el patrón de consumo que proporciona tanto al paciente como al terapeuta un dato de referencia para posteriores comparaciones durante el proceso de abandono.

- Terapia sustitutiva asociada en forma de parches transdérmicos de nicotina, según del grado de dependencia, de $17,5,35$ y $52,5 \mathrm{~m} / \mathrm{g}$ y comprimidos de $2 \mathrm{~m} / \mathrm{g}$ durante dos meses aplicada, con disminución paulatina, a todos los participantes que lo precisan ${ }^{11}$. Esta decisión se toma sopesando muy diversos factores: el nivel de consumo (puntuación obtenida en el test de Fargerstron), la situación medico-sanitaria del sujeto y la propia demanda del usuario. (Hay que tener en cuenta los casos en que la medicación sustitutiva se encuentra contraindicada por embarazo, padecimiento coronario o de otra índole, donde juega un importante papel el equipo sanitario del centro).

E) Creación de un sistema motivacional de retroalimentación, del modo siguiente:

${ }^{10}$ Barrueco Ferrero M. Manual de prevención y tratamiento del tabaquismo. Madrid, 2003.

11 Ídem anterior. 


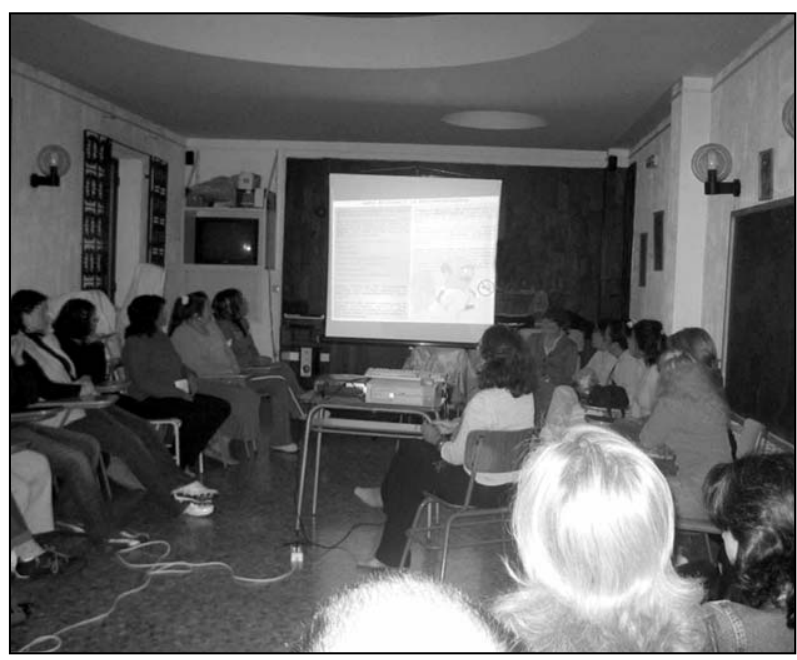

Grupo de terapia de deshabituación tabáquica.

- Asistencia conjunta de trabajadores e internas a las sesiones de apoyo personal, donde se trabajan los avances (y eventuales recaídas), en un plano de igualdad, lo que favorece el papel terapéutico. La medición pública de los niveles de CO también es un factor muy determinante para el refuerzo de los periodos de abstinencia, sobre todo en los primeros meses, donde la dependencia física es más intensa. (Ej: "si mi educador lo consigue, no bay razón para que no lo consiga yo... lo demostraré.” “...estaría bueno que la mayoría de las internas tenga más fuerza de voluntad que yo").

- Puesta en marcha de una estrategia global de apoyo y refuerzo, donde el resto del personal penitenciario alienta individualmente a cada uno de los componentes del grupo, en los lugares donde desempeñan su actividad diaria (reforzando su asistencia a actividades deportivas, formativas y ocupacionales); y con menciones públicas cada vez que lo permite algún acto de relevancia: actuaciones, teatro, etc.

En esta última etapa, la intervención propiamente dicha, se establece una perfecta coordinación entre los profesionales del centro (ya formados en las técnicas del programa), y los técnicos externos. La coordinación corre a cargo de la Subdirectora de Tratamiento, con el apoyo logístico del personal del centro penitenciario: monitor deportivo ${ }^{12}$, psicóloga y educadores. El personal sanitario se ocupa de dispensación de tratamientos directamente observados

${ }^{12}$ Daniel González, de la ONG BATA, que por convenio con la Junta de Andalucía desarrolla su actividad en este establecimiento, y de quien partió la idea inicial de este programa.
(TDO) y del control sanitario de las internas participantes.

\section{OBJETIVOS OPERATIVOS}

En relación a los resultados de la encuesta de consumo del centro, antes de iniciar la primera edición del programa se establecieron unos objetivos operativos que nos permitieran evaluar los resultados finales de la intervención. En esta previsión de resultados se valoró el peso negativo que debía ejercer la significativa prevalencia de consumo y los condicionantes negativos que acompañan a la situación de encarcelamiento.

1. Abandono total del hábito tabáquico al final del programa (4 meses) de un porcentaje del $15 \%$ de las mujeres y/o trabajadores que lo inician (en el medio libre, el porcentaje de abandono del hábito tabáquico mediante terapia multicomponente se sitúa alrededor del $30-40 \%)^{13}$.

2. Persistencia de la abstinencia tabáquica al cabo de un año desde el inicio del programa del $10 \%$ de las personas que participaron en el mismo.

3. Disminución del $5 \%$ del consumo diario de cigarrillos de las mujeres y/o trabajadores que siguen el programa, aunque sin conseguir el abandono definitivo del hábito tabáquico.

4. Permanencia del $60 \%$ de internas y/o trabajadores en el programa hasta el final de duración del mismo.

$5.25 \%$ de nuevas solicitudes de participación en la siguiente edición anual del programa de aquellos integrantes que no consiguieron abandonar el consumo de cigarrillos en su primer intento. Éste es un dato que avala la carga motivacional adquirida hacia futuros intentos de deshabituación.

6. Disminución de la venta de tabaco en el economato del centro penitenciario (es el único punto de acceso a tabaco de las internas) al primer mes del inicio del programa, como medida indirecta del impacto de su implantación.

7. Aumento del número de solicitudes para participar en la segunda edición del programa respecto a la primera.

En la fase de inicio de la segunda edición del programa, estos objetivos se adaptaron a los resultados reales de esta primera experiencia, de tal forma que el objetivo operativo global era alcanzar resultados similares a los obtenidos en la primera edición.

\footnotetext{
${ }^{13}$ Según se refiere en Mateos Vilchez P. Tratamiento grupal multicomponente. Ver nota $\mathrm{n}^{\circ} 8$.
} 


\section{RESULTADOS}

\section{ÍNDICES DE EVALUACIÓN}

\section{PRIMERA EDICIÓN: AÑO 2005-2006}

- Solicitudes para participar en la primera edición del programa de deshabituación tabáquica: 25 solicitudes de internas y 2 de trabajadores.

- Número de internas y/o trabajadores seleccionados para iniciar el programa (septiembre 2005): 22 internas y 2 trabajadores.

- Número de internas y/o trabajadores que finalizan el programa (diciembre 2005): 19 internas (86,4\%) y 2 trabajadores $(100 \%)$.

- Internas y/o trabajadores que han abandonado el hábito tabáquico a fecha de finalización del programa (diciembre 2005): 9 internas $(40,9 \%$ ) y 2 trabajadores (100\%).

- Persistencia del no consumo al cabo de un año (diciembre 2006): 5 internas (22,7\%) y 2 trabajadores $(100 \%)$.

- Consumo medio de cigarrillos de las personas que siguieron el programa y que no han abandonado definitivamente el hábito tabáquico: no se ha medido, aunque se estima (por declaraciones subjetivas) que puede encontrarse alrededor del 50\%.

- Disminución de la venta de tabaco en el economato general del centro (es el único punto de acceso de las internas) al mes del inicio de los programas: $15,13 \%$, entre septiembre y octubre de 2005 (de $5.457,55$ a $4.631,70 €)$.

\section{SEGUNDA EDICCIÓN: 2006-2007}

- Número de solicitudes para participar en la segunda edición del programa de deshabituación tabáquica: 35 peticiones de internas y 6 de trabajadores (en diembre 2006).

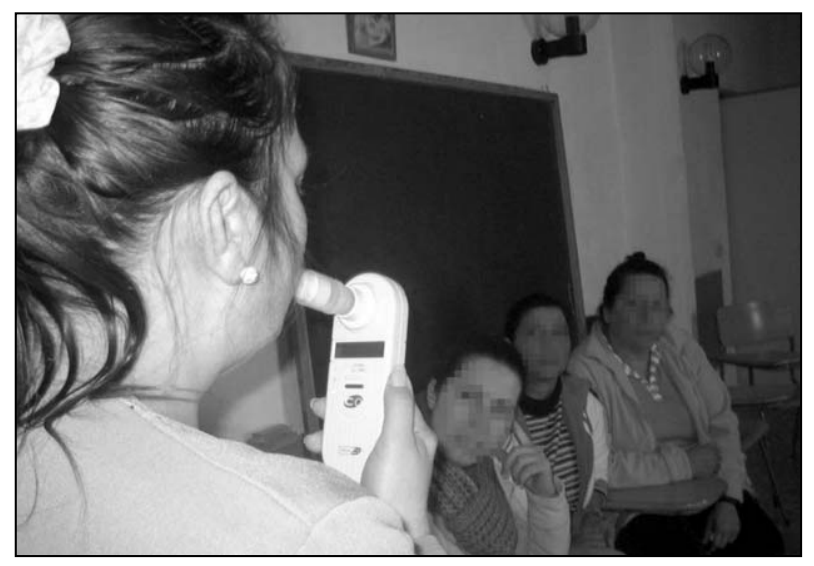

Medición de los niveles de CO mediante cooximetro.

- Número de internas y/o trabajadores seleccionados para iniciar el programa: 22 internas y 3 trabajadores.

- Porcentaje de personas que, tras haber finalizado la primera edición del programa (año 2006) pero sin éxito en el abandono del consumo de cigarrillos y permaneciendo en el centro, solicitan su participación en la nueva edición anual del programa (año 2006-2007): el 50\% (3 internas de las 6 que siguen destinadas en el centro). No se valora el dato de trabajadores al ser nulo el índice de fracaso.

- Disminución de la venta de tabaco en el economato general del centro al mes del inicio de los programas: 13,00\% entre octubre y noviembre de 2006 (de $6.574,30$ a $5.719,55 €)$.

\section{DISCUSIÓN DE RESULTADOS}

Los resultados de nuestro programa han estado muy por encima de las expectativas iniciales que el equipo responsable del mismo tenía, tanto respecto al abandono del hábito tabáquico entre las internas y/o trabajadores, como en relación a la persistencia de la

\section{Participantes al inicio del programa}

\section{Abandono del tabaco a la finalización del programa} (4 meses)
Persistencia del no consumo de tabaco al año de inicio del programa

\begin{tabular}{|c|c|c|c|c|c|c|}
\hline PRIMERA EDICIÓN & Internas: & 22 & Internas: & $9(40,9 \%)$ & Internas: & $5(22,7 \%)$ \\
\hline$(2005-2006)$ & Trabajadores: & 2 & Trabajadores: & $2(100 \%)$ & Trabajadores: & $2(100 \%)$ \\
\hline $\begin{array}{l}\text { SEGUNDA EDICIÓN } \\
(2006-2007)\end{array}$ & $\begin{array}{l}\text { Internas: } \\
\text { Trabaiad }\end{array}$ & $\begin{array}{r}20 \\
3\end{array}$ & $\begin{array}{l}\text { Internas: } \\
\text { Trabaiado }\end{array}$ & $\begin{array}{l}12(60 \%) \\
3(100 \%)\end{array}$ & $\begin{array}{l}\text { Internas: } \\
\text { Trabaiadores: }\end{array}$ & $\begin{array}{r}6(30 \%) \\
3(100 \%)\end{array}$ \\
\hline
\end{tabular}




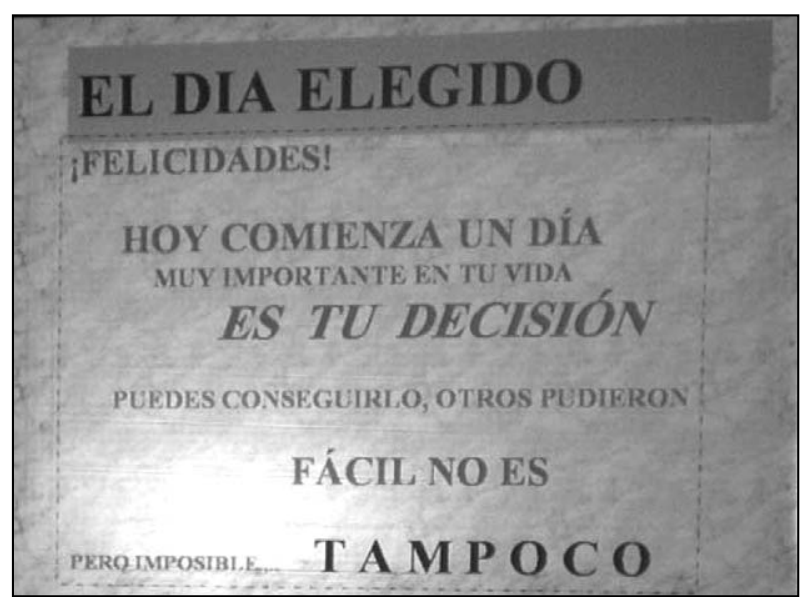

Panel de trabajo correspondiente a la sesión del día " $D$ "

deshabituación a lo largo del periodo de año y medio transcurrido desde el inicio del programa.

En nuestra opinión, la campaña previa motivacional y el propio desarrollo del programa facilitaron la ausencia de incidentes durante la implantación, al 1 de enero de 2006, de la Ley 28/2005 antitabaco ${ }^{14}$, aunque para poder valorar apropiadamente este dato es necesario hacer una comparación con otras prisiones de características similares que no contaron con este programa de deshabituación.

Hemos observado un cambio en la cultura institucional, donde el no fumar, o abandonar el hábito de fumar adquiere valor y connotaciones positivas. Esto, por el momento, es una valoración subjetiva que planeamos ilustrar en un futuro próximo mediante una encuesta que compare la cultura institucional respecto al tabaco en nuestro centro con otras prisiones sin programa de deshabituación al tabaco.

A través de nuestro programa hemos conseguido implicar a las autoridades sanitarias y penitenciarias. Esto contribuye a cohesionar la prisión y también a estrechar la colaboración de nuestro centro con los centros sanitarios de la provincia tanto en el nivel de atención primaria (área de salud del Distrito sanitario de Alcalá de Guadaíra) como en la atención especializada (Hospital de Valme). Del éxito del programa se beneficia también el resto de la atención sanitaria y no sanitaria de la prisión.

\section{CONCLUSIONES}

- Hemos conseguido demostrar, en la práctica, que es posible implantar y generalizar con éxito programas

${ }^{14}$ Ley 28/2005, de 26 de diciembre, de medidas sanitarias frente al tabaquismo y reguladora de la venta, el suministro, el consumo y la publicidad de los productos del tabaco. de abandono de la dependencia del tabaco en los Establecimientos penitenciarios. Esto supone un paso importante en la mejora de la salud global de los internos que sufren una mayor prevalencia de tabaquismo que la población general y de los trabajadores penitenciarios, que comparten con ellos un espacio reducido y cerrado.

- Consideramos que por su escasa complejidad, y por suponer un coste económico asumible y eficiente, el periodo de permanencia en un centro penitenciario puede ser un momento adecuado para iniciar y consolidar un intento serio de abandono del hábito de fumar.

- Consideramos que, en nuestra experiencia, la implicación de los trabajadores penitenciarios como participantes activos en los programas de desintoxicación y deshabituación ha sido un elemento decisivo y diferencial del éxito de esta intervención.

- El éxito de este programa en el Centro Penitenciario de mujeres de Alcalá de Guadaíra es un ejemplo a utilizar para que el hábito del tabaco entre a formar parte ordinaria de los planes de tratamiento de drogodependencias y otras adicciones y se implante en la generalidad de los establecimientos penitenciarios.

\section{ASPECTOS FORMATIVOS}

Para el mantenimiento de esta actividad es imprescindible la colaboración con programas comunitarios de salud especializados en la deshabituación tabáquica, que posibiliten que nuestro personal pueda adquirir los conocimientos y destrezas necesarios para asumir paulatinamente este cometido. Esta colaboración seguirá siendo relevante en la formación continua de nuestro personal y en el apoyo logístico: avances, material, etc.

Esta experiencia muestra que la colaboración intersectorial en el abordaje de los problemas de salud en las poblaciones desfavorecidas es viable y eficaz.

\section{REPERCUSIÓN SUPRAINSTITUCIONAL}

Desde 1995 la Organización Mundial de la Salud (OMS) puso en marcha el Proyecto de Salud en las Prisiones (HIPP) para promocionar la salud en la comunidad penitenciaria, impulsando el intercambio de información y buenas prácticas de salud, la elaboración de recomendaciones y la difusión de la experiencia acumulada a lo largo de los años. 
Siguiendo estos objetivos, en 2003 se acordó la creación de un premio europeo bianual para destacar las buenas prácticas de salud en las prisiones. España se incorporó al Proyecto de Salud en las Prisiones en el 2004. Actualmente, 32 estados europeos forman parte de la red. El premio tiene como objetivo la identificación, el reconocimiento y la difusión de los mejores programas y políticas en materia de promoción de la salud y prevención de la enfermedad en instituciones penitenciarias y la utilidad del trabajo para su aplicación en otros países miembros de la OMS.

Convencidos de la importancia de esta experiencia, presentamos un resumen de este proyecto en la edición del año 2007, en la categoría no 2 , como "Ejemplo de Buena práctica relacionado con uno o varios de los siguientes servicios ofrecidos a los internos: de prevención, de educación en salud, y de promoción de la salud". En esta edición el "Programa de deshabituación tabáquica para internos y trabajadores de un centro penitenciario" del Centro Penitenciario de Mujeres de Alcalá de Guadaíra (Sevilla) recibió el premio en Trencin, Slovakia (noviembre 2007).

http://www.uclan.ac.uk/facs/health/hsdu/settings/ who awards 2007.html

http://www.uclan.ac.uk/old/facs/health/hsdu/ settings/documents/awards2007/Cat\%202\%20Spain \%20Alcala.pdf

\section{REFERENCIAS BIBLIOGRÁFICAS}

1. Barrueco-Ferrero M. Manual de prevención y tratamiento del tabaquismo. Madrid 2003.

2. Cropsey K, Eldridge G. Smoking among female prisioners: An ignorerd public health epidemic. Addictive Behaviors 29 (2004) 425-431

3. Delegación del Gobierno para el Plan Nacional sobre Drogas. Encuesta de salud de instituciones penitenciarias 2006.

4. Mateos-Vilchez P. Tratamiento grupal multicomponente. Red de Formadores del Plan Integral de Atención al Tabaquismo de Andalucía. www. redformadorespita.org

5. Stead LF, Lancaster T. Group behaviour therapy programmes for smoking cessation (Cochrane Review 2002/2005). The Cochrane Library, Issue 2. Oxford: Update Software.

6. Sieminska A. Prisoners'attitudes towards cigarette smoking and smoking cessation: a questionnarire study in Poland. BMC Public Health 2006 jul 7; 6: 181 .

\section{CORRESPONDENCIA}

Talleres Editoriales Cometa, S.A.

Ctra. de Castellón, km. 3,400

50013 Zaragoza 